Document downloaded from:

http://hdl.handle.net/10251/64684

This paper must be cited as:

Sirci, S.; Martínez Pérez, JD.; Vague Cardona, JJ.; Boria Esbert, VE. (2015). Substrate Integrated Waveguide Diplexer Based on Circular Triplet Combline Filters. IEEE Microwave and Wireless Components Letters. 25(7):430-432. doi:10.1109/LMWC.2015.2427516.

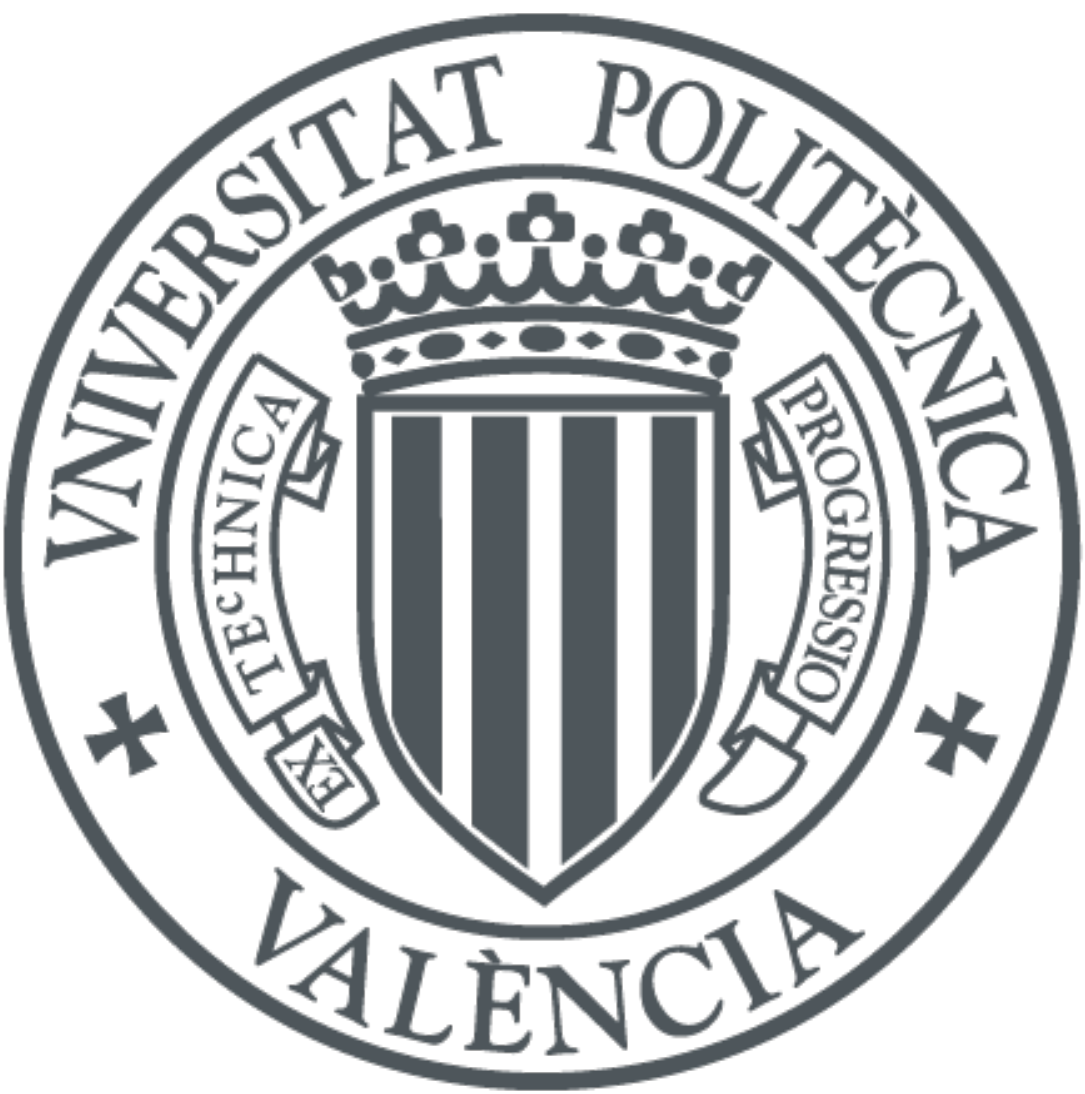

The final publication is available at

http://dx.doi.org/10.1109/LMWC.2015.2427516

Copyright Institute of Electrical and Electronics Engineers (IEEE)

Additional Information 


\title{
Substrate Integrated Waveguide Diplexer Based on Circular Triplet Combline Filters
}

\author{
Stefano Sirci, Student Member, IEEE, Jorge D. Martínez, Member, IEEE, \\ Joaquín Vague, and Vicente E. Boria, Senior Member, IEEE
}

\begin{abstract}
The design of substrate integrated waveguide (SIW) diplexers, based on combline triplet sections with transmission zeros (TZs) placed below and above the passband, is presented. In order to control the location of the TZs, positive and negative couplings are conveniently provided. A highly compact implementation based on circular substrate integrated coaxial resonators is proposed. An $\mathrm{X}$-band diplexer with channel center frequencies at 9.5 and $10.5 \mathrm{GHz}$ and absolute bandwidths of $400 \mathrm{MHz}$ is designed. The structure shows important advantages in terms of size reduction, while keeping good insertion losses as well as high rejection and isolation levels.
\end{abstract}

Index Terms - Combline filters, diplexer, substrate integrated waveguide (SIW), transmission zeros (TZs).

\section{INTRODUCTION}

D IPLEXERS are essential components in transceivers for microwave communication systems, in order to effectively separate transmit and receive channels connected to a common antenna. Because they have a great impact on the overall system performance, low insertion losses, strong channel rejection and high isolation are usually required.

Substrate integrated waveguide (SIW) is a well-known alternative in order to reach a trade-off solution between waveguide and planar technologies in terms of quality factor, volume, power handling and easy integration. Thus, several diplexers have been recently proposed in SIW technology. In [1] a C-band diplexer is proposed based on inductive SIW filters obtaining moderate insertion losses but large occupied area. On the other hand, a highly miniaturized SIW diplexer is proposed in [2] reaching low insertion loss, although channel rejection and isolation are limited due to the achieved response even for a large separation between channels. Therefore, the aforementioned alternatives present some limitations for implementing compact diplexers with high performance and minimum channel spacing.

Recently, substrate integrated coaxial resonators have been proposed for reducing filter size in SIW technology [3]. In this paper, both positive and negative cross-couplings in a circular triplet section are presented in order to achieve high rejection

This work was supported by MINECO (Spanish Government) under projects TEC2103-47037-C5-1-R and TEC2013-48036-C3-3-R.

S. Sirci and V. E. Boria are with the iTEAM, Universitat Politècnica de València, València E-46022, Spain.

J. D. Martínez is with the I3M, Universitat Politècnica de València, València E-46022, Spain (e-mail: jdmartinez@eln.upv.es).

J. Vague is with the Departamento de Ingeniería Electrónica, Universitat Politècnica de València, València E-46022, Spain.

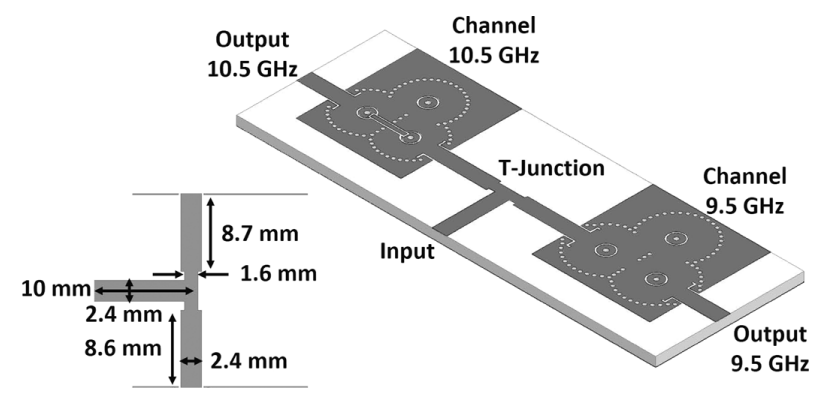

Fig. 1. Scheme of the proposed diplexer using substrate integrated triplets in combline topology.

in compact X-band diplexers with narrow inter-channel separation. It is shown that electric coupling can be easily introduced in a single side of the structure, thus alleviating problems derived from the common approach in conventional SIW filters that require slots at both sides of the substrate [4]. Thus, the device can be fabricated using low cost single-layer PCB processes, and good agreement has been obtained between simulation and measurements demonstrating the proposed approach.

\section{DIPLEXER}

\section{A. Structure}

The diplexer structure is shown in Fig. 1. It is composed of two channel filters connected to a common input port through a microstrip T-junction. The filters are independently designed using three-pole circular substrate integrated coaxial resonators [5]. Coupling level to each filter is provided through a CPW current probe with two insets that enables to fix the input/output external quality factor.

As can be seen in Fig. 2, each filter consists on three interleaved substrate integrated coaxial resonators. A plated via hole at the center of the resonator is used as the inner conductor. The outer conductor is defined by the SIW via wall. The inner conductor is short-circuited at the bottom and capacitively loaded at the top. The loading capacitance can be created using a circular slot around the inner conductor at the top metal layer of the SIW. In order to improve rejection, the lower-band channel introduces a TZ at the upper-side. This can be done using a positive magnetic coupling between resonators 1 and 3 . On the contrary, the upper-band filter introduces a TZ at the lower-side by means of a negative electric coupling between them. While positive coupling is provided using inductive irises between adjacent resonators, the negative one is generated through coupling strips entering the loading capacitive disk.

A low-cost 1.524 mm-thick PCB substrate with $\epsilon_{r}=3.55$ and $\tan \delta=2.7 \cdot 10^{-3}$ has been used for implementing an 


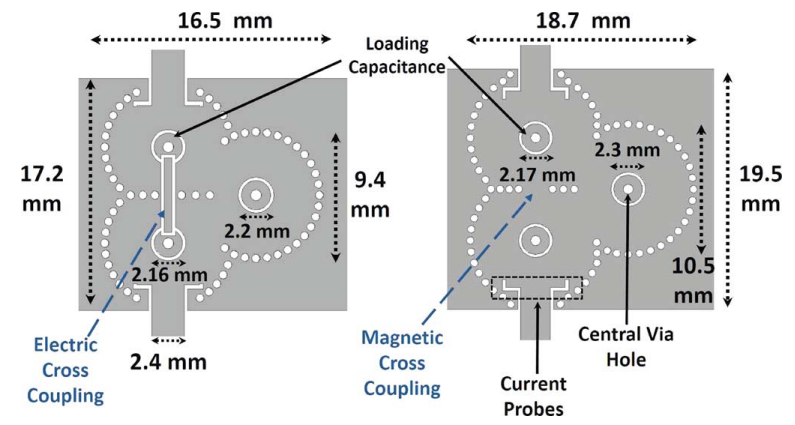

Fig. 2. Detail of the channel filters with negative (left) and positive (right) cross-coupling between resonators 1 and 3 .

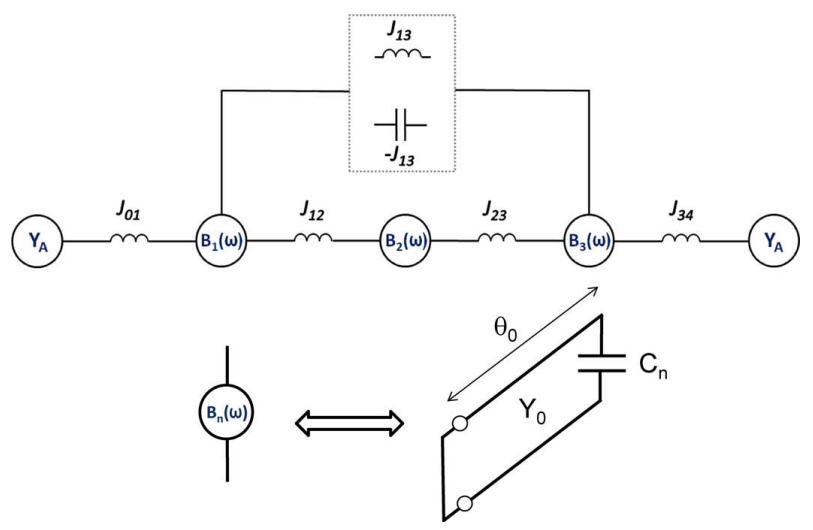

Fig. 3. Equivalent circuit of the substrate integrated combline triplet.

$\mathrm{X}$-band prototype with channel filters centered at 9.5 and $10.5 \mathrm{GHz}$, respectively, and $400 \mathrm{MHz}$ bandwidth. The TZs are located at 10.2 and $9.8 \mathrm{GHz}$ in order to provide high rejection level at the opposite channel band.

\section{B. Filter Synthesis}

The equivalent circuit of each triplet is shown in Fig. 3. The resonator can be seen as a TEM-mode combline resonator [3]. The synthesis procedure starts by choosing the slope parameter $b$ of the basic cell at the center frequency of the filter. Then, the impedance of the coaxial resonator, and the ratio between the inner via $d_{v}$ and the outer via wall $2 \cdot r_{\mathrm{SIW}}$ diameter, is obtained from the expression of the susceptance slope parameter and the impedance of a circular-circular coaxial transmission line as

$$
\begin{aligned}
Z_{0} & =\frac{\cot \theta_{0}+\theta_{0} \csc ^{2} \theta_{0}}{2 b} \\
\frac{2 \cdot r_{\mathrm{SIW}}}{d_{v}} & =0.9268 \cdot \exp ^{Z_{0} \sqrt{\epsilon_{r}} / 60} .
\end{aligned}
$$

Thus, a slope parameter $b=20 \mathrm{mS}$ has been chosen for both filters. The physical length for all resonators is always the same one (i.e., corresponding to the substrate thickness). However, in terms of electrical lengths, the values are $\theta_{0, l}=$ $32.73^{\circ}$ and $\theta_{0, u}=36.18^{\circ}$ for the lower and upper channel filters respectively. This gives a coaxial resonator impedance of $Z_{0, l}=87.75 \Omega$ and $Z_{0, u}=79.48 \Omega$, corresponding to $\left(2 \cdot r_{\mathrm{SIW}} / d_{v}\right)_{l}=14.6$ and $\left(2 \cdot r_{\mathrm{SIW}} / d_{v}\right)_{u}=11.24$.

Then, a circuit-level optimization of the filter is performed in order to obtain the capacitance of each resonator and the inverter values. An initial guess considering an under-coupled cross-coupling and synchronous resonators is used. A symmetrical filter topology is also forced during this procedure. The

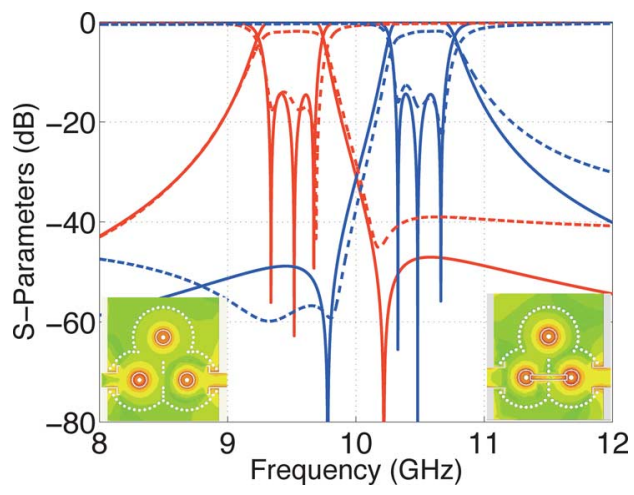

Fig. 4. Synthesized (solid) and simulated (dashed) $S_{11}$ and $S_{21}$ parameters of the diplexer channel filters.

loading capacitance values for the lower and upper filter are $C_{1, l}=C_{3, l}=0.283 \mathrm{pF}, C_{2, l}=0.270 \mathrm{pF}, C_{1, u}=C_{3, u}=$ $0.273 \mathrm{pF}, C_{2, l}=0.276 \mathrm{pF}$. Identically, the inverter values are given by $J_{01, l}=J_{34, l}=3.65 \cdot 10^{-3} \mathrm{~S}, J_{12, l}=J_{23, l}=6.51$. $10^{-4} \mathrm{~S}, J_{13, l}=1.64 \cdot 10^{-4} \mathrm{~S}, J_{01, u}=J_{34, u}=3.62 \cdot 10^{-3} \mathrm{~S}$, $J_{12, u}=J_{23, u}=6.50 \cdot 10^{-4} \mathrm{~S}$, and $J_{13, u}=-1.49 \cdot 10^{-4} \mathrm{~S}$. The responses of the synthesized channel filters are shown in Fig. 4.

\section{Resonators and Couplings Dimensioning}

Filter layout can be obtained from the former optimized equivalent circuit of each channel. Starting from the basic resonator cell, inner via diameter is fixed as $d_{v}=0.8 \mathrm{~mm}$ and the distance from inner via to SIW wall $r_{\mathrm{SIW}}$ can be easily derived given the ratios $\left(2 \cdot r_{\mathrm{SIW}} / d_{v}\right)$. The capacitance value is fixed by changing the radius of the annular gap $g$ for a particular resonant frequency.

Input and output couplings are controlled by changing the dimensions of the current probes as shown in Fig. 5. For a given set of probe dimensions, the desired level of the external quality factor $Q_{e x t}=b Y_{A} / J_{01}^{2}=b Y_{A} / J_{34}^{2}$ can be fixed by modifying the penetration $l_{i n 2}$ of the coplanar probe inside the cavity. Positive coupling is achieved by means of inductive windows between adjacent resonators. The width of the iris enables to control the inter-resonator coupling coefficient $k_{i j}$ as shown in Fig. 5. On the other hand, electrical coupling is achieved using an open-ended capacitive probe located between resonators 1 and 3 at the top side of the substrate. This probe passes through an iris window created between the shared resonator wall of the non-contiguous resonators. Due to the mixed character of this coupling, the electrical or magnetical contribution can be controlled by changing the probe penetration and/or the iris window as it is shown in Fig. 6.

The design procedure starts with the values obtained from the equivalent circuit and using the former design curves for $Q_{e x t}$ and $k_{i j}$, followed by a final refinement using 3-D fullwave analysis. Simulated results of the designed channel filters are also depicted in Fig. 4, showing a good agreement with the previously synthesized responses.

\section{T-Junction Design}

A simple T-junction has been designed for the common port. Thus, an open-circuit condition has been forced at the junction for the opposite channel filter in order to improve isolation and minimize channel filter interaction. Therefore, given the input 
TABLE I

COMParison TABLE Between ReFERENCES AND PROPOSEd DipleXer

\begin{tabular}{|c|c|c|c|c|}
\hline Ref. & Size & Insertion Loss $(\mathrm{dB})$ & Rejection $(\mathrm{dB})$ & Isolation \\
\hline$[1]$ & $2.71 \lambda_{0} \times 3.35 \lambda_{0} \times 0.009 \lambda_{0}$ & $2.6 \mathrm{~dB} / 3.2 \mathrm{~dB}$ & $55 \mathrm{~dB} @ 5.42 \mathrm{GHz} / 55 \mathrm{~dB} @ 5.96 \mathrm{GHz}$ & $53 \mathrm{~dB}$ \\
\hline$[2]$ & $0.27 \lambda_{0} \times 0.22 \lambda_{0} \times 0.008 \lambda_{0}$ & $1.6 \mathrm{~dB} / 2.3 \mathrm{~dB}$ & $43 \mathrm{~dB} @ 4.66 \mathrm{GHz} / 28 \mathrm{~dB} @ 5.80 \mathrm{GHz}$ & $32 \mathrm{~dB}$ \\
\hline$[6]$ & $1.44 \lambda_{0} \times 0.98 \lambda_{0} \times 0.022 \lambda_{0}$ & $2.2 \mathrm{~dB} / 2.4 \mathrm{~dB}$ & $22 \mathrm{~dB} @ 7.75 \mathrm{GHz} / 22 \mathrm{~dB} @ 8.25 \mathrm{GHz}$ & - \\
\hline This work & $2.04 \lambda_{0} \times 0.65 \lambda_{0} \times 0.053 \lambda_{0}$ & $1.6 \mathrm{~dB} / 2.1 \mathrm{~dB}$ & $42 \mathrm{~dB} @ 9.50 \mathrm{GHz} / 37 \mathrm{~dB} @ 10.50 \mathrm{GHz}$ & $35 \mathrm{~dB}$ \\
\hline
\end{tabular}
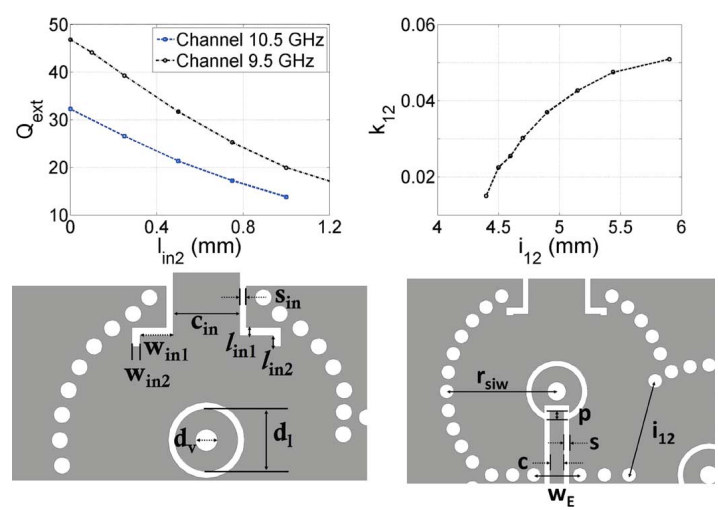

Fig. 5. $Q_{\text {ext }}$ and positive $k_{i j}$ as a function of the coplanar current probe length inside the cavity $l_{i n 2}$ and the iris width $i_{i j}$ respectively.

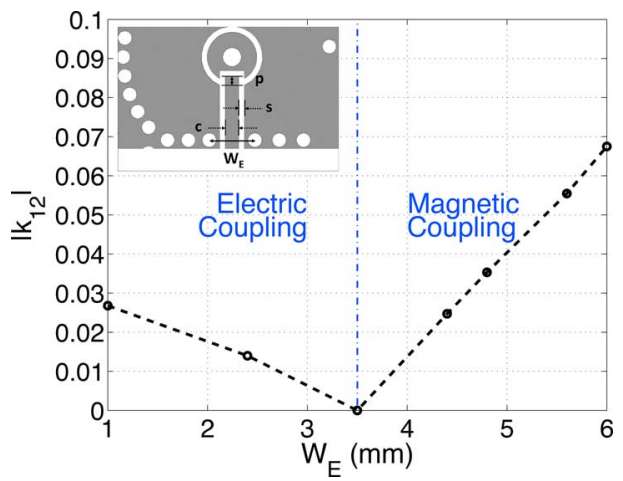

Fig. 6. Inter-resonator coupling magnitude as a function of the cross-coupling iris width. The mixed coupling is dominated by the electrical contribution for a given probe penetration $p$ when the iris width is reduced.

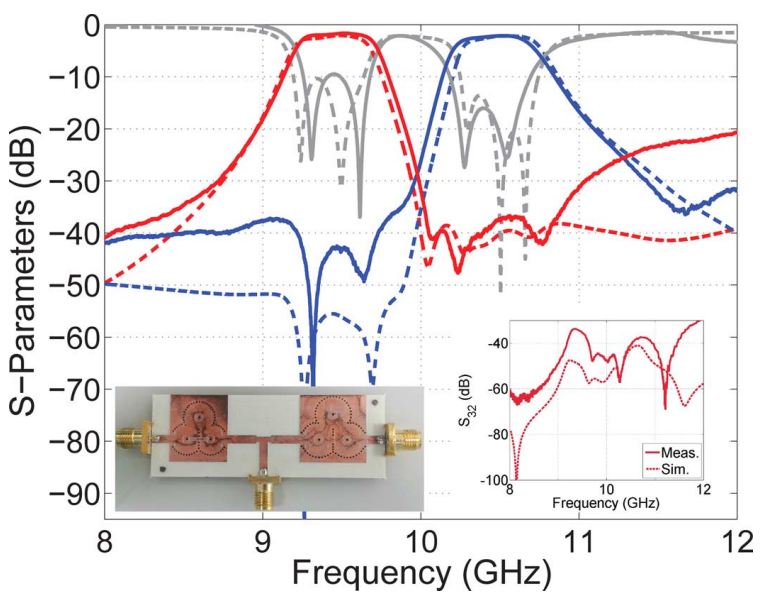

Fig. 7. Measured (solid) and simulated (dashed) $S_{21}, S_{31}$ and $S_{11}$ paramaters of the diplexer. Isolation between channels $S_{32}$ is also shown in the subfigure.

impedance $Z_{i n,\{l, u\}}(f)$ of each filter seen from the junction reference plane, a section of transmission line has been added in order to force $Z_{i n, l}\left(f_{0, u}\right)=\infty$ and $Z_{i n, u}\left(f_{0, l}\right)=\infty$, while keeping the matching condition at the center frequency of each channel filter. Finally, a small step in width on the microstrip T-junction ports has been added for compensating the direct discontinuity. Lastly, the T-junction dimensions have been optimized at circuital level without any change on the independent channel filters design. The dimensions of the T-junction are also shown in Fig. 1.

\section{RESULtS}

The diplexer has been manufactured in $1.524 \mathrm{~mm}$-thick Rogers $4003 \mathrm{C}$ with $17 / 17 \mu \mathrm{m} \mathrm{Cu}$ clad using a standard single-side fabrication process. A photo of the fabricated device is shown in Fig. 7. The whole device dimensions are 58.4 $\times 18.7 \mathrm{~mm}^{2}$ excluding the microstrip feeding lines.

Simulated and measured results are shown in Fig. 7 showing a very good agreement. As can be seen, an additional TZ is obtained at the upper-channel filter due to the cancellation of the electric and magnetic contribution of the mixed coupling between non-adjacent resonators. Minimum insertion losses are 1.6 and $2.1 \mathrm{~dB}$ at the lower and upper channels respectively, while return loss at the common port is better than $10 \mathrm{~dB}$ in both cases. Stopband suppresion for both channels is better than $37.5 \mathrm{~dB}$, while a minimum isolation of $35 \mathrm{~dB}$ has been obtained. Table I presents a comparison between some other designs (i.e., [1], [2], [6]) and the proposed structure. Good performance can be obtained in terms of losses, rejection and isolation even for almost contiguous channels, while showing reasonable size compared to other SIW structures.

\section{CONCLUSION}

A compact SIW diplexer based on substrate integrated combline triplets is proposed and demonstrated at $X$-band. High rejection and isolation even for closely spaced channels is obtained by introducing TZs at the lower or upper band of each filter. A simple and low-cost single-side PCB process can be used for manufacturing this component.

\section{REFERENCES}

[1] Z. C. Hao, W. Hong, J. X. Chen, X. P. Chen, and K. Wu, "Planar diplexer for microwave integrated circuits," Proc. Inst. Elect. Engi., vol. 152 , no. 6 , pp. $455-459$, Dec. 2005.

[2] Y. Dong and T. Itoh, "Substrate integrated waveguide loaded by complementary split-ring resonators for miniaturized diplexer design," IEEE Microw. Wireless Compon. Lett., vol. 21, no. 1, pp. 10-12, Jan. 2011.

[3] J. D. Martínez, S. Sirci, M. Taroncher, and V. E. Boria, "Compact CPW-fed combline filter in substrate integrated waveguide technology," IEEE Microw. Wireless Compon. Lett., vol. 22, no. 1, pp. 7-9, Jan. 2012.

[4] X.-P. Chen and K. Wu, "'Substrate integrated waveguide cross-coupled filter with negative coupling structure," IEEE Trans. Microw. Theory Tech., vol. 56, no. 1, pp. 142-149, Jan. 2008.

[5] J. D. Martínez, S. Sirci, and V. E. Boria, "Compact SIW filter with asymmetric frequency response for C-band wireless applications," in Proc. Int. Wireless Symp., Beijing, China, Apr. 2013, pp. 1-4.

[6] A. García-Lampérez, M. Salazar-Palma, and S.-H. Yeung, "SIW compact diplexer," in IEEE MTT-S Int. Dig., Jun. 2014, pp. 1-4. 